\title{
On the Continuous Limit of Integrable Lattices I. The Kac-Moerbeke System and KdV Theory
}

\author{
Carlo Morosi ${ }^{1}$, Livio Pizzocchero ${ }^{2}$ \\ ${ }^{1}$ Dipartımento di Matematica, Politecnıco di Milano, Piazza L da Vinci 32, I-20133 Milano, Italy \\ 2 Dipartimento dı Matematica, Università dı Mılano, Via C Saldini 50, I-20133 Milano, Italy and \\ Istituto Nazıonale di Fisica Nucleare, Sezıone di Milano, Italy \\ E-mail: pizzocchero@vmımat mat unimi it
}

Received: 10 October 1995/Accepted: 4 March 1996

\begin{abstract}
KdV}$ theory is constructed systematically through the continuous limit of the Kac-Moerbeke system The infinitely many commuting vector fields, the conserved functionals, the Lax pairs and the biHamiltonian structure are recovered as the limits of suitably defined linear combinations of homologous objects for the Kac-Moerbeke system The combinatorial aspects of this recombination method are treated in detail
\end{abstract}

\section{Introduction}

In the literature about integrable lattices, one meets the statement that the KdV-type equations can be obtained as continuous limits of the evolution equations of suitably chosen nonlinear lattices After the pioneering work of Zabusky and Kruskal [ZK] on the continuous limit of the Fermi-Pasta-Ulam lattice, Toda pointed attention to the integrable lattice theories giving the $\mathrm{KdV}$ (or the Boussinesq) equation in the continuous limit The limit process for the Toda lattice (from now on indicated with the acronym TL) was first described by Toda and Wadati [TW], this was the beginning of an effort, aiming to get more insight on the relation between discrete integrable systems and $\mathrm{KdV}$-type theories The second edition of Toda's book [Tod] quotes, for example, Saitoh's papers [Sai], other references will be given in the sequel

The discussion of the continuous limit has a counterpart in the realm of inverse scattering The papers of Case, Chiu and Kac [CCK] are a classical reference on this topic in the discrete version of inverse scattering presented here, the limit process is easily performed at any step of the construction, yielding to the standard theory for the Schrödinger spectral problem

Leaving aside the inverse scattering, and coming to the structural analysis of the integrable evolution equations, we cannot avoid quoting Kupershmidt's monograph [Kup] Here a general, purely algebraic setting is proposed for the integrable lattices, extending to discrete systems the techniques for integrable KdV-type field theories developed by the Russian school [Dic]; after constructing a formal variational 
calculus and a theory of differential-difference operators, Kupershmidt discusses in this framework the limit case in which the lattice spacing goes to zero

In this paper we are interested, in particular, in the ordinary KdV theory as a continuous limit of the so-called Kac-Moerbeke system This is a reduction of the usual TL (from now on denoted with the acronym KM), if $a=\left(a_{l}\right)$ and $b=\left(b_{i}\right)$ are the standard Flaschka coordinates for TL ( $i$ being the index for lattice sites), then the phase space of the KM system is the submanifold defined by the constraint $b_{i}=0$ (see Sect 2 for the necessary details) The system was considered in [KM], where the angle-action coordinates were constructed, it was also discussed by Zakharov, Musher, Rubenchik and Manakov [ZMR, Man] The KM Lax operator is the differential-difference operator already considered in the papers [CCK] on inverse scattering

The fact that the KM evolution equation gives the ordinary KdV equation in the continuous limit was understood early (as reported in [Sch], Kac obtained the KdV one-soliton solution from the limit process) The convergence of the KM solutions to $\mathrm{KdV}$ solutions, for arbitrary initial conditions, was proved rigorously by Schwarz in the quoted reference

In spite of the above mentioned relevant results on the correspondence between $\mathrm{KM}$ and $\mathrm{KdV}$ theory, we think that there is still an opportunity to say something new on this topic We list a number of problems which, in our opinion, are essentially open

i) Most of the literature aims to recover from the continuous limit of the KM system the $\mathrm{KdV}$ evolution equation

$$
u_{\tau}=u_{u}+6 u u_{r}
$$

and/or its Lax formulation

$$
\frac{d L^{\mathrm{KdV}}}{d \tau}=\left[B_{2}^{\mathrm{KdV}}, L^{\mathrm{KdV}}\right]
$$

where $L^{\mathrm{KdV}}(u)=\hat{\imath}_{u}+u$ and $B_{2}^{\mathrm{KdV}}(u)=4 \hat{c}_{m}+6 u \hat{c}_{1}+3 u_{1}$. (Here, we denote by $\tau$ the evolution parameter, reserving the letter $t$ for the KM system ) On the other hand, it is known that the vector field $\left(\begin{array}{ll}1 & 1\end{array}\right)$ is a member of an infinite hierarchy of commuting vector fields, it would be interesting to obtain all these fields, and the related Lax pairs, through a systematic limit procedure on the KM hierarchy

ii) Of course, one would like to obtain in the same scheme the full sequence of $\mathrm{KdV}$ conserved functionals

iii) The $\mathrm{KdV}$ and Toda-type hierarchies are biHamiltonian each vector field has two different Hamiltonian descriptions, given in terms of two compatible Poisson tensors It would be interesting to recover the $\mathrm{KdV}$ biHamiltonian structure as a continuous limit In the quoted book, Kupershmidt discusses the limit of Poisson structures on a lattice, even though his treatment allows to obtain the first $\mathrm{KdV}$ Poisson tensor, it is not clear how to recover the second one Indeed, should one interpret too naively some statement of the author ( conclusion that the Poisson structures arising as continuous limits of a lattice theory contain only the first power of the operator $\hat{c}_{x}$, this would exclude the possibility to recover the second $\mathrm{KdV}$ Poisson tensor, which is a third order differential operator in $\hat{c}_{1}$ 
In this paper, we propose a solution for problems i), ii) and iii). Our derivation of $\mathrm{KdV}$ theory from the KM system is based on a systematic method, which can be as well applied to other systems, starting from a different lattice, we plan to recover the Boussinesq theory in a subsequent paper

The biHamiltonian formalism plays a central role in our construction, for this reason, our attitude is to define the $\mathrm{KM}$ and $\mathrm{KdV}$ systems in biHamiltonian terms, and to introduce subsequently the Lax formalism, we take advantage from this viewpoint in order to prove that the continuous limit can be performed for the whole KM hierarchy

In order to set up the limit process for the KM system, we interpolate the sequence $\left(a_{1}\right)$ of phase space coordinates with a smooth function $a(x)$ of a continuous variable $x$, and introduce a rescaled field variable $u$ related to $a$ by

$$
a(x)=2+\varepsilon^{2} u(x),
$$

$\varepsilon$ being the lattice spacing This is, essentially, the variable change considered in [Kup] to obtain Eq $\left(\begin{array}{ll}1 & 1\end{array}\right)$ and the $\mathrm{KdV}$ Lax operator in the $\varepsilon \mapsto 0$ limit

The distinctive feature of our approach is a recombination principle which allows to construct the full $K d V$ theory (the infinitely many vector fields and conserved functionals, the Poisson tensors, the Lax pairs) taking the limits of some suitable chosen linear combinations of homologous objects for the KM system. We anticipate here the main results arising through the application of this method, the proofs will be given in Sect 5 (Propositions 5 4-6)

Let $f_{i}(u)(s=0,1,2, \quad)$ be the sequence of conserved functionals for the KM system, given in terms of the $u$ variable on account of the transformation (1 3 ), similarly, let $X_{s}^{\prime}(u)(s=1,2,3, \quad)$ be the $\mathrm{KM}$ vector fields (see Sects 3 and 4) For each $s$ we form the linear combinations

$$
h_{s}^{i}(u)=f_{\zeta}^{\xi}(u)+\sum_{j=0}^{i-1} c_{\varsigma,} f_{j}^{\xi}(u)-c_{\varsigma}, \quad Z_{\zeta}^{\xi}(u)=X_{\varsigma}^{\xi}(u)+\sum_{j=1}^{s-1} c_{s,} X_{j}^{\xi}(u),
$$

where $c_{\triangleleft j}$ and $c_{s}$ are numerical coefficients determined by an explicit prescription (see Sect 5) Our result is that the zero-spacing limits of the $h_{s}$ and $Z_{s}^{s}$ are the conserved functionals and the vector fields of order $s$ of the $\mathrm{KdV}$ hierarchy

$$
\lim _{\varepsilon \rightarrow 0} \frac{h_{\varsigma}^{\varepsilon}(u)}{4^{\varsigma} \varepsilon^{2 s+2}}=h_{\varsigma}^{\mathrm{KdV}}(u), \quad \lim _{\varepsilon \mapsto 0} \frac{Z_{s}^{\sharp}(u)}{4^{s+1} \varepsilon^{2 \uparrow-1}}=Z_{\varsigma}^{\mathrm{KdV}}(u)
$$

Even in the case of the $\mathrm{KdV}$ equation (11) (i.e, the vector field $Z_{2}^{\mathrm{KdV}}$ in our notations), the derivation based on $\mathrm{Eq}$ (15) turns out to be different from the derivation given in [Kup], this point will be discussed in the Remark at the end of Sect. 4

In our approach, the above results can be derived from the $\varepsilon \mapsto 0$ limit of the KM biHamiltonian recursion scheme, the limit of the biHamiltonian structure can also be treated by means of a recombination In fact, let $Q_{u}^{\varepsilon}$ and $P_{u}^{\varepsilon}$ be the Poisson tensors of the KM system, we will show that $Q_{u}$ and $S_{u}^{c}=P_{u}^{u}-16 Q_{u}$ give respectively, for $: \mapsto 0$, the first and the second KdV Poisson tensors

$$
\lim _{\varepsilon \mapsto 0} \frac{1}{4} \varepsilon^{3} Q_{u}^{\varepsilon}=Q_{u}^{\mathrm{KdV}}, \quad \lim _{i: 0} \frac{1}{16} c S_{u}^{\varepsilon}=S_{u}^{\mathrm{KdV}}
$$

The same limit technique can be applied to the Lax pairs For each $s$, let us consider the $\mathrm{KM}$ vector field $X_{s}^{*}$ and its Lax formulation $d L^{*} / d t_{s}=\left[A_{s}^{i}, L^{\xi}\right]$, to recover from 
here the KdV Lax pairs $d L^{\mathrm{KdV}} / d \tau_{s}=\left[B_{s}^{\mathrm{KdV}}, L^{\mathrm{KdV}}\right]$ we will consider the operators

$$
B_{\zeta}^{\prime}(u) .=A_{i}^{\prime}(u)+\sum_{j=1}^{i-1} c_{s,} A_{j}^{i}(u)
$$

and show that

$$
\lim _{\varepsilon: 0} \frac{L^{\varepsilon}(u)-4}{2 \varepsilon^{2}}=L^{\mathrm{KdV}}(u), \quad \lim _{\varepsilon \mapsto 0} \frac{B_{s}(u)}{4^{s+1} \varepsilon^{2 s-1}}=B_{\varsigma}^{\mathrm{KdV}}(u)
$$

One could be tempted to formulate the above results in a more picturesque style, using the language of renormalization well known from field theory [GJ] We confine this temptation to the next few lines, and dare to say that the lattice spacing $\delta$ is a cut-off, while $a(x)$ and $u(x)=\frac{a(x)-2}{\varepsilon^{2}}$ are the "bare" and "renormalized" fields. The recombination scheme for the functionals $f^{\prime}$ somehow recalls the Wick product in our model one gets the wanted $\delta \mapsto 0$ limit replacing $f_{s}$ with $h_{s}^{\varepsilon}=f_{s}^{\varepsilon}+$ (a linear combination of the $f_{j}^{\prime}$, s for $j=0,1,, s-1$ ) + a constant, whereas in constructive field theory one operates on the action functional and replaces the powers $\phi^{\prime}$ of the field variable $\phi$ with the Wick powers . $\phi^{\varsigma}$, each of them being expressible as $\phi^{s}+$ (a linear combination of the lowest order powers $\left.\phi^{j}\right)+$ a constant

The paper is organized as follows In Sect 2, we review the basic facts on the KM system (also giving its second Poisson structure, which does not seem to be well known in the literature), in the same section, we recall how to obtain the KM system as a reduction of TL, and we fix our notational standards about KdV theory In Sect 3, we discuss the interpolation of the discrete KM system in terms of continuous field variables In Sect 4 , we introduce the recombination method and the zero-spacing limit procedure In Sect 5 , we give the general proofs of the statements (1 5), (1 6) and (1.8) As an exercise, in Sect 6 we show that the one-soliton solutions for the evolution equations of the first three recombined vector fields $Z_{s}$ converge for $\varepsilon \mapsto 0$ to the one-soliton solutions of the first three vector fields $Z_{\mathrm{s}}^{\mathrm{KdV}}$, here, we also include some remarks on the general problem of the $\varepsilon \mapsto 0$ limit for the flows of the recombined hierarchy Some of the computations presented in Sects 2, 4 have been performed using the MATHEMATICA symbolic manipulation system Sects $2-5$ of the paper could well be written in the merely algebraic language of formal variational calculus, but we prefer to adopt a less abstract presentation, a formulation of our results in the very pure style of [Kup] will be given elsewhere

\section{Preliminaries: Kac-Moerbeke Versus KdV}

In this section, we work with spaces of real sequences $p=\left(p_{l}\right)_{l \in \mathcal{Z}}$, where $\mathscr{F}$ is the set of integers $\mathbb{Z}$ (infinite case) or $\mathscr{Z}=\mathbb{Z}_{N}=\mathbb{Z} / N \mathbb{Z}$, with $N$ any integer (periodic case) When necessary, suitable boundary conditions will be assumed in the infinite case concerning the behaviour of sequences for $i \rightarrow \pm \infty$ If $p$ is a sequence and $r \in \mathscr{Z}, p_{(l)}$ is the $r$-shifted sequence of elements $p_{(l) t}=p_{t+1}{ }^{1}$ The shift operator sending $p$ into $p_{(1)}$ is denoted by $\Delta$, obviously, the power $\Delta^{\prime}$ sends $p$ into $p_{(\prime)}$

\footnotetext{
' On the contraly, we denote by $p^{\prime}$ the ${ }^{\text {th }}$ power of the sequence $p$, with elements $\left(p^{\prime}\right)_{l}=\left(p_{l}\right)^{\prime}$
} 
If we consider the real sequences over $\mathscr{Z}$ as an algebra with the pointwise product $(p q)_{l}=p_{i} q_{l}$, then $\Delta$ turns out to be an algebra automorphism. For any fixed sequence $g$ and each integer $r$, composing the shift and the multiplication operator by $g$ we can form the operators $g \Delta^{\prime}$ and $\Delta^{\prime} g$, these act on any sequence $\psi=\left(\psi_{l}\right)_{l \in \mathcal{Z}}$ according to the rules $\left(g \Delta^{\prime}\right)(\psi)=g \psi_{(l)}$, $\left(\Delta^{\prime} g\right)(\psi)=g_{(1)} \psi_{(1)}$, and we have $\Delta^{\prime} g=g_{(1)} \Delta^{\prime}$ If a second sequence $h$ is fixed, by composition we can construct the operator $g \Delta^{\prime} h$, and so on

Following [Kup], one can also introduce the associative algebra $\mathfrak{g}$ of formal pseudo differential-difference operators

$$
G=\sum_{i=-\infty}^{\prime \max } y_{1} \Delta^{\prime}
$$

where each coefficient $y_{1}$ is a sequence over $\mathscr{Z}, r_{\max }$ is an arbitrary integer (depending on $G$ ) This algebra carries a trace if the coefficient $y_{0}$ in Eq (2 1) is a sequence with elements $g_{0, i}$, then $\operatorname{Tr} G=\sum_{t \in \boldsymbol{y}} g_{0, i}$

2 I The Kac-Moerbeke $(K M)$ system The phase space of the system is a conveniently chosen set $\mathscr{A}$ of real sequences $a=\left(a_{l}\right)_{i \in \mathcal{Z}}$ We denote by $T_{a} \mathscr{A}$ and $T_{a}^{*} \mathscr{A}$ the tangent and cotangent spaces at any point $a$, their elements are represented as real sequences, written as $\dot{a}=\left(\dot{a}_{l}\right)$ and $\dot{\delta} a=\left(\delta a_{l}\right)$ respectively We have the pairing $\langle\delta a, \dot{a}\rangle=\sum_{l \in y} \delta a_{l} \dot{a}_{i}$

The manifold carries a biHamiltonian structure, i.e, a pair of Poisson tensors $Q, P$ which are mutually compatible in the sense of Magri [Mag] At each point $a$, we have $Q_{a} \quad T_{a}^{*} \mathscr{A} \rightarrow T_{a} \mathscr{A}, \delta a \mapsto \dot{a}=\frac{1}{2} a\left(a_{(1)} \delta a_{(1)}-a_{(-1)} \delta a_{(-1)}\right)$, or, in operator form,

$$
\dot{a}=\frac{1}{2}\left(a \Delta a-a \Delta^{-1} a\right)(\delta a)
$$

The second tensor $P_{a} \quad T_{a}^{*} \cdot \mathscr{d} \rightarrow T_{a} \cdot \mathscr{A}$ sends a covector $\delta a$ into

$$
\dot{a}=\frac{1}{2}\left(a^{3} \Delta a-a \Delta^{-1} a^{3}+a \Delta a^{3}-a^{3} \Delta^{-1} a+a \Lambda a^{2} \Delta a-a \Delta^{-1} a^{2} \Delta^{-1} a\right)(\delta a)
$$

An infinite hierarchy of commuting vector fields $X_{\varsigma}(s=1,2,3, . \quad)$ and conserved functionals $f_{s}(s=0,1,2, \quad)$ can be defined recursively on \& using the biHamiltonian structure $Q, P$ The starting point is the function

$$
f_{0}(a)=\sum_{l \in \boldsymbol{Z}} \log a_{l}
$$

which is a Casimir of $Q \cdot Q d f_{0}=0$ The subsequent functionals and the vector fields of the hierarchy are defined by means of the recursion relations

$$
X_{s+1}=Q d f_{\mathrm{s}+1}=P d f_{s} \quad(s=0,1,2, \quad)
$$

If $f_{s}$ is known, the above equation gives $X_{s+1}$ and fixes $f_{s+1}$ up to the addition of a Casimir of $Q$ (i e, $f_{0}$ or an arbitrary function of $f_{0}$ ), from the functional form of $f_{0}$, it is evident that the ambiguity in $f_{s+1}$ can be removed with the following prescription $f_{s+1}$ must be the sum over $i$ of a polynomial expression in $a_{i}, a_{i \pm 1}, a_{i \pm 2}$, including no constant term Using this algorithm, 
one finds

$$
\begin{gathered}
f_{1}(a)=\sum_{i \in \mathcal{y}} a_{l}^{2}, \quad f_{2}(a)=\sum_{l \in \mathcal{y}}\left(\frac{1}{2} a_{l}^{4}+a_{l}^{2} a_{l+1}^{2}\right), \\
X_{1}(a)=a\left(a_{(1)}^{2}-a_{(-1)}^{2}\right), \\
X_{2}(a)=a\left(a_{(1)}^{4}-a_{(-1)}^{4}+a^{2} a_{(1)}^{2}-a^{2} a_{(-1)}^{2}+a_{(1)}^{2} a_{(2)}^{2}-a_{(-1)}^{2} a_{(-2)}^{2}\right), \\
X_{3}(a)=a\left(a_{(1)}^{6}-a_{(-1)}^{6}+a^{4} a_{(1)}^{2}-a^{4} a_{(-1)}^{2}+2 a^{2} a_{(1)}^{4}-2 a^{2} a_{(-1)}^{4}\right. \\
+a_{(1)}^{2} a_{(2)}^{4}-a_{(-1)}^{2} a_{(-2)}^{4}+2 a_{(1)}^{4} a_{(2)}^{2}-2 a_{(-1)}^{4} a_{(-2)}^{2}+a^{2} a_{(1)}^{2} a_{(2)}^{2} \\
\left.-a^{2} a_{(-1)}^{2} a_{(-2)}^{2}+a_{(1)}^{2} a_{(2)}^{2} a_{(3)}^{2}-a_{(-1)}^{2} a_{(-2)}^{2} a_{(-3)}^{2}\right),
\end{gathered}
$$

and so on

Equation (25) tells us that each vector field $X_{s+1}$ of the KM hierarchy is Hamiltonian with respect to both $Q$ and $P$, the Hamiltonian functions being $f_{\mathrm{s}+1}$ and $f$, respectively As it is known from the standard theory of biHamiltonian systems, this implies that the functionals $f$, are conserved quantities for all vector fields of the hierarchy, and they are in involution for both the $Q$ and $P$ Poisson brackets, moreover, the vector fields $X_{\varsigma}$ mutually commute

One can also produce a Lax formulation of the KM hierarchy, resting on the Lax operator

$$
L(a)=\Delta^{-1} a+a \Delta
$$

If $d / d t$, denotes the Lie derivative along the vector field $X_{\varsigma}$, we have

$$
\frac{d L}{d t_{\varsigma}}=\left[A_{\varsigma}, L\right], \quad A_{\varsigma}=\left(L^{2 \varsigma}\right)_{\mathrm{skcw}},
$$

where, for each $G$ as in Eq (21), $G_{\text {skew }}$ denotes the skew-symmetric part of $G$, given by $G_{\text {skeu }}=\sum_{l=1}^{I_{\text {max }}}\left(g_{1} \Delta^{\prime}-\Delta^{-\prime} g_{l}\right)$ Equation (29) can be as well employed in place of the biHamiltonian recursion (2 5) to define the vector fields $X$, For $s=1,2,3$, the Hamiltonians $f$, can also be characterized in terms of the Lax operator, since

$$
f_{s}(a)=\frac{1}{2 s} \operatorname{Tr} L^{2 s}(a)
$$

22 The KM system as a reduction of $T L$ The relation between the two systems is apparent in the Lax approach, and a bit more subtle from the biHamiltonian viewpoint To clarify this point, it is convenient to review some facts about TL

We systematically use the Flaschka variables [Fla]. The phase space $\mathscr{I}$ of TL is a set of pairs $m=(a, b)$, where $a$ and $b$ are $\mathscr{Z}$-indexed real sequences, at each point $m, T_{m} . / /$ and $T_{m}^{*}$. $/ /$ denote respectively the tangent and the cotangent spaces, whose elements are represented as pairs $m=(\dot{a}, b)$ and $\delta m=(\delta a, \delta b)$, with the pairing $\langle\delta m, \dot{m}\rangle=\sum_{l \in \dot{y}}\left(\delta b_{l} b_{l}+\delta a_{l} \dot{a}_{l}\right)$ Two compatible Poisson tensors $P_{1}$ and $P_{2}$ are defined on $\mathscr{K}$, at each point $m$ we have $P_{1 m} \quad T_{m}^{*}: \| \rightarrow T_{m} \cdot \mathscr{H}, \dot{\delta} m \mapsto \dot{m}=P_{1 m} \delta m$, with $\dot{a}=a\left(\delta b_{(1)}-\delta b\right)$ and $b=a \delta a-a_{(-1)} \delta a_{(-1)}$, or, in matrix form,

$$
\left(\begin{array}{l}
\dot{a} \\
b
\end{array}\right)=\left(\begin{array}{cc}
0 & a(\Delta-1) \\
\left(1-\Delta^{-1}\right) a & 0
\end{array}\right)\left(\begin{array}{l}
\delta a \\
\dot{\delta} b
\end{array}\right)
$$


Similarly, $P_{2 m}$ sends a covector $(\delta a, \delta b)$ into the tangent vector

$$
\left(\begin{array}{l}
\dot{a} \\
b
\end{array}\right)=\left(\begin{array}{cc}
\frac{1}{2} a\left(\Delta-\Delta^{-1}\right) a & a(\Delta-1) b \\
b\left(1-\Delta^{-1}\right) a & 2 a^{2} \Delta-2 \Delta^{-1} a^{2}
\end{array}\right)\left(\begin{array}{l}
\delta a \\
\delta b
\end{array}\right) .
$$

An infinite hierarchy of commuting vector fields $x^{\prime}(s=1,2,3, \quad)$ and Hamiltonians $\varphi,(s=0,1,2, \quad)$ can be constructed recursively on .// starting from

$$
\varphi_{0}(m)=\sum_{l \in \mathscr{Z}} \log a_{l}, \quad \varphi_{1}(m)=\sum_{l \in \mathscr{Z}} b_{l} ;
$$

$\varphi_{0}$ is a Casimir of both Poisson tensors $P_{1}$ and $P_{2}$, while $\varphi_{1}$ is a Casimir of $P_{1}$ The higher order Hamiltonians and the vector fields of the hierarchy are defined stipulating the recursion relation

$$
\mathscr{x}_{\mathrm{s}}=P_{2} d \varphi_{\mathrm{s}}=P_{1} d \varphi_{\mathrm{s}+1} \quad(s=1,2,3, \quad) .
$$

For each $s$, the above equation determines $\varphi_{s+1}$ from $\varphi_{\text {s }}$ up to the addition of a Casimir of $P_{1}$ The nature of the Casimirs allows to remove this ambiguity with the following prescription $\varphi_{s+1}$ must be (the sum over $i$ of) a polynomial in $a_{l}, b_{l}$ and their shifts of any order $a_{l+1}, b_{l+l^{\prime}}$, including neither linear terms in $b$ nor constant terms In particular, the first vector field

$$
x_{1}(m)=\left(\begin{array}{c}
a\left(b_{(1)}-b\right) \\
2\left(a^{2}-a_{(-1)}^{2}\right)
\end{array}\right)
$$

gives, in the Flaschka variables, the evolution equations for the chain of exponential springs first considered by Toda

All vector fields of the TL hierarchy admit a Lax formulation in terms of the Lax operator

$$
\Lambda(m)=\Delta^{-1} a+b+a \Delta
$$

If $d / d t$, denotes the derivative along $\mathscr{*}$, we have

$$
\frac{d \Lambda}{d t_{s}}=\left[\left(\Lambda^{\mathrm{s}}\right)_{\mathrm{skcu}}, \Lambda\right]
$$

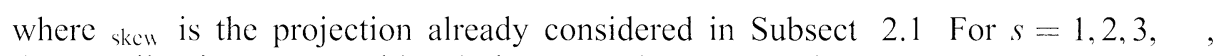
the Hamiltonians $\varphi$, considered above can be expressed as

$$
\varphi_{s}(m)=\frac{1}{s} \operatorname{Tr} \Lambda^{`}(m)
$$

Now, let us observe that the TL Lax operator $\Lambda(m)$ becomes the KM operator $L(a)$ if one sets $b=0$ in Eq (216) From a geometrical viewpoint, this amounts to consider in the phase space.// the submanifold formed by pairs $m=(a, 0)$, this submanifold can be identified with the phase space $S$ of the KM system, and it can be shown that the geometrical structures considered in Subsect 21 are restrictions to of homologous structures living on II

Let us start from the biHamiltonian formalism The TL Poisson tensor $P_{1}$ cannot be restricted ${ }^{2}$ to $\mathscr{S}$, conversely, $P_{2}$ restricts into the Poisson tensor $Q$ of Eq (2 2)

\footnotetext{
${ }^{2}$ For a leview on the restriction techniques of Poisson tensors, see for example [MMR]
} 
In order to recover the second tensor $P$ of the KM system, we must consider, besides $P_{1}$ and $P_{2}$, the higher order TL Poisson structures These can be introduced using the recursion operator $N_{m}=P_{2 m} \circ P_{1 m}^{-1}$ (where the inverse of $P_{1}$ is understood in the framework of formal pseudo differential-difference operators) At each point $m=(a, b)$, we have

$$
N_{m}=\left(\begin{array}{cc}
(a b-a \Delta b)(a-a \Delta)^{-1} & \frac{1}{2}(a+a \Delta) \\
2\left(\Delta^{-1} a^{2}-a^{2} \Delta\right)(a-a \Delta)^{-1} & b
\end{array}\right)
$$

By means of $N$, one can define the tensors $P_{s}=N^{s-2} P_{2}(s=3,4,5, \quad)$, which form (together with $P_{1}$ and $P_{2}$ ) a sequence of mutually compatible Poisson structures $^{3}$ It turns out that $N^{2}$ restricts from $\mathscr{U}$ to $\mathscr{A}$, this implies restrictability of $P_{4}=N^{2} P_{2}$, which goes into the KM Poisson tensor $P$ of Eq (2 3)

Using the reducibility of $N^{2}$, one can show that all even order vector fields $x_{2 s}$ of the TL hierarchy are tangent to the submanifold $\mathscr{A}$, for $s=1,2,3$, the restrictions of the $X_{2}$, are just the KM vector fields $X$, Similarly, the restrictions to .f of the Hamiltonians $\varphi_{2}$, are the KM Hamiltonians $f$, (the odd order Hamiltonians $\left(\varphi_{2 s+1}\right.$ are identically zero on $\left.\mathscr{A}\right)$

23 A glossary of $K d V$ theory In order to fix the notations, we add a few lines about $\mathrm{KdV}$ theory Here the phase space is a set $\|$ of real smooth functions $u$ of a variable $x$, ranging over the real line $\mathbb{R}$ or the torus $\mathbb{T}=\mathbb{R} / \mathbb{Z}$ Elements of the tangent space $T_{u} \|$ and the cotangent space $T_{\|}^{*} \|$ are smooth functions of $x$, denoted typically by $u$ and $\delta u$, we have the pairing $\left.\langle\dot{\delta} u, \dot{u}\rangle=\int d x \dot{\delta}\right\rangle(x) \dot{u}(x)$ The geometrical and algebraic structures attached to the phase space $\mathbb{N}$ will be marked with the letters $\mathrm{KdV}$, in order to avoid confusions in the rest of the paper We have two Poisson tensors $Q^{\mathrm{KdV}}, S^{\mathrm{KdV}}$, at each point $u, Q_{u}^{\mathrm{KdV}} . T_{u}^{*}\left\|\mapsto T_{u}\right\|$ sends a covector $\delta u$ into the tangent vector

$$
u=\delta u_{\imath}
$$

while $S_{u}^{\mathrm{KdV}}$ sends $\delta u$ into

$$
u=\left(\hat{c}_{u}+4 u \hat{c}_{1}+2 u_{1}\right)(\dot{\partial} u)
$$

We denote with $Z_{\varsigma}^{\mathrm{KdV}}(s=1,2,3,$.$) and h_{\varsigma}^{\mathrm{KdV}}(s=0,1,2, \quad)$ the vector fields and the Hamiltonians of the familiar $\mathrm{KdV}$ hierarchy, these can be defined recursively starting from

$$
h_{0}^{\mathrm{KdV}}(u)=\frac{1}{2} \int d x u(x)
$$

(which is a Casimir of $Q$ ) and stipulating that

$$
Z_{\varsigma+1}^{\mathrm{KdV}}=Q^{\mathrm{KdV}} d h_{\varsigma+1}^{\mathrm{KdV}}=S^{\mathrm{KdV}} d h_{\varsigma}^{\mathrm{KdV}} \quad(s=0,1,2, .),
$$

with the supplementary requirement that $h_{s+1}^{\mathrm{KdV}}$ be (the integral over $x$ of) a polynomial in $u$ and its derivatives, containing neither linear terms in $u$ nor constant terms. For example $Z_{1}^{\mathrm{KdV}}(u)=u_{1}, Z_{2}^{\mathrm{KdV}}(u)$ is the $\mathrm{rhs}$ of Eq $(11), h_{1}^{\mathrm{KdV}}(u)=\frac{1}{2} \int d x u^{2}$, $h_{2}^{\mathrm{KdV}}(u)=\int d x\left(u^{3}-\frac{1}{2} u_{1}^{2}\right)$, etc

\footnotetext{
${ }^{3}$ This sequence could also be constructed via the so-called "mastersymmetry approach," see e g $[\mathrm{Dam}]$
} 
Of course, this hierarchy can be described in terms of the Lax operator

$$
L^{\mathrm{KdV}}(u)=\hat{c}_{x}+u
$$

If $d / d \tau$, is the derivative along $Z_{\varsigma}^{\mathrm{KdV}}$, we have

$$
\frac{d L^{\mathrm{KdV}}}{d \tau_{\varsigma}}=\left[B_{\varsigma}^{\mathrm{KdV}}, L^{\mathrm{KdV}}\right], \quad B_{\varsigma}^{\mathrm{KdV}}=4^{\varsigma-1}\left(\left(L^{\mathrm{KdV}}\right)^{\varsigma-\frac{1}{2}}\right)_{+},
$$

where + denotes the projection on the nonnegative powers of $\partial_{1}$ in the algebra of formal pseudo differential operators, furthermore,

$$
h_{\varsigma}^{\mathrm{KdV}}(u)=\frac{4^{\varsigma}}{2 s+1} \operatorname{Tr}\left(L^{\mathrm{KdV}}\right)^{s+\frac{1}{2}}(u),
$$

where $\operatorname{Tr}$ is the well known Adler traceform carried by this algebra [Dic]

\section{Interpolating the KM System}

The constructions of Sect. 2 arise from an abstract algebraic structure, which can be described as follows we have a commutative algebra (the sequences over $\mathscr{Z}$, with the pointwise product), carrying a distinguished automorphism (the shift operator $\triangle$ ) and a linear functional (the sum $\sum_{i \in \mathcal{y}}$ ) invariant under this automorphism There is an obvious, alternative realization of the same abstract structure, where the sequences are replaced by real smooth functions of a continuous variable $x$, ranging over the real line $\mathbb{R}$ or the torus $\mathbb{T}$. In the present section we consider this alternative realization, the system arising in this way can be easily interpreted as an interpolation of the discrete KM lattice of Sect 2 (see the final Remark) and provides the starting point to recover $\mathrm{KdV}$ theory through an appropriate limit process

Let us consider the algebra of real smooth functions $a$ on $\mathbb{R}$ or $\mathbb{T}$ (with appropriate boundary conditions at infinity in the former case) We fix a fundamental spacing $\varepsilon \in \mathbb{R}$, the shift operator on smooth functions is the map $\Delta_{\xi} a \mapsto a_{(\xi)}$, where $a_{(i)}(x)=a(x+\varepsilon)$ For each integer $r$, the $r^{\text {th }}$ power of $\Delta_{i}$ is the operator sending $a$ into $a_{(i \varepsilon)}$, where $a_{(i \varepsilon)}(x)=a(x+r \varepsilon)$, this operator will be denoted by $\Delta_{i:}$ The linear functional $\left(a_{l}\right) \mapsto \sum_{l \in \mathcal{Z}} a_{t}$ on sequences is replaced in the present framework by the functional $a \mapsto \int d x a(x)$ (integration over the real line or the torus), which is clearly shift invariant 4

If $g$ and $h$ are fixed smooth functions, we can form operators such as $g \Delta_{i}$, $\Delta_{i} g, g \Delta_{i} h$, for example, the third one acts on a function $\psi$ with the rule $\left(g \Delta_{r} h\right)(\psi)(x)=g(x) h(x+r \varepsilon) \psi(x+r \varepsilon)$ Also, we can introduce an algebra of formal pseudo differential-difference operators $G_{i}=\sum_{l=-\infty}^{I_{\max }} g_{l} \Delta_{l \varepsilon}$, where the coefficients $y$, are smooth functions of $x$ This is clearly the counterpart of the algebra $\mathfrak{g}$ in Sect 2, it carries a traceform $\operatorname{Tr}$, where $\operatorname{Tr} G_{\varepsilon}=\int d x g_{0}(x)$

\footnotetext{
${ }^{4}$ In the case whete $r$ ranges over the line $\mathbb{R}$, it is necessary for our purposes to define the integral $f d a(1)$ for each function of the form $a(x)=c+l(1)$, with $c$ a constant and $l$ vanishing rapidly at infinity If $a$ is of this form, we put $\int d x a(x):=\int d x v(x)$ ("subtraction of the vacuum infinities"); according to this prescription, for each constant $c$ it is $\int d x c=0$ An analogous definition could well be given for $\sum_{l} a_{l}$, when $i$ ranges over the set $\mathbb{Z}$ of integers and $\left(a_{l}\right)$ is the sum of a constant and a rapıdly vanıshing sequence
} 
We now come to the interpolated KM system, its construction rephrases Subsect 22, so it is hardly the case to mention the essential points Concerning the notations, our style will be to maintain the same letters for the homologous objects in the discrete and continuous cases, adding the mark $\&$ to any structure which depends on the spacing

So, the phase space of the interpolated system will be denoted by $\mathscr{A}$, its elements are real smooth functions $a$ on $\mathbb{R}$ or $\mathbb{T}$ The pairing between cotangent vectors $\delta a$ and tangent vectors $\dot{a}$ is given by $\langle\delta a, \dot{a}\rangle=\int d x \delta a(x) \dot{a}(x)$. We have a pair of Poisson tensors $Q^{\varepsilon}, P^{\varepsilon}$, defined by formulas analogous to (2.2), (2 3 ) (provided that we systematically replace the symbols $a_{(\prime)}$ and $\Delta^{\prime}$ with $a_{(1 \varepsilon)}$ and $\left.\Delta_{t:}\right)$, the recursion relation

$$
X_{s+1}^{\delta}=Q^{s} d f_{s+1}^{c}=P^{i} d f_{s}^{\vdots}, \quad(s=0,1,2, \quad)
$$

gives rise to a hierarchy of vector fields $X_{s}^{\varepsilon}$ and Hamiltonians $f_{s}^{\xi}$, starting from

$$
f_{0}^{g}(a)=\int d x \log a(x)
$$

(in fact, $s$ independent) For $s=1,2,3$, , we stipulate that $f_{s}$ be the integral over $x$ of a polynomial in $a(x)$ and the shifts $a_{(r)}(x)$, including no constant term, with this supplementary requirement, the recursion formula (3 1 ) uniquely determines $f_{s+1}^{\xi}$, if $f_{s}$ is known The first Hamiltonians after $f_{0}^{i}$ are

$$
f_{1}(a)=\int d x a^{2}(x), \quad f_{2}(a)=\int d x\left(\frac{1}{2} a^{4}(x)+a^{2}(x) a^{2}(x+\varepsilon)\right)
$$

The explicit expressions for the vector fields $X_{1}^{i}, X_{2}^{i}$ and $X_{3}^{i}$ are analogous to formulas (2 7), with the obvious prescription of replacing $a_{(1)}$ with $a_{(1,)}$

The hierarchy has a Lax operator $L^{*}(a)=a \Delta_{i}+\Delta_{i}^{-1} a$; Eqs (2 9) and (2 10) are still satisfied, i e,

$$
\frac{d L^{\varepsilon}}{d t_{\mathrm{s}}}=\left[A_{\varsigma}^{\varepsilon}, L^{\varepsilon}\right], \quad A_{\varsigma}^{\varepsilon}=\left(\left(L^{\varepsilon}\right)^{2 \varsigma}\right)_{\mathrm{skcw}}
$$

and

$$
f_{s}^{\varepsilon}(a)=\frac{1}{2 s} \operatorname{Tr}\left(L^{i}\right)^{2 s}(a),
$$

intending the trace as explained above

Remark Let us add some comments on the relation between the interpolated and the discrete KM systems Of course, the discrete index $i$ of Sect 2, ranging over $\mathscr{Z}$, can be viewed as the label for a network of equally spaced sites on $\mathbb{R}$ or $\mathbb{T}$, of coordinates $x_{l}=i \varepsilon$ If $\mathscr{Z}=\mathbb{Z}$, the points $x_{i}$ lie on the line and $\varepsilon$ is arbitrary, if $\mathscr{Z}=\mathbb{Z}_{N}$, the $N$ points $x_{l}$ are placed on the torus, with $\varepsilon= \pm 1 / N$

Given a smooth function $a$ of $x$ and a sequence $\left(a_{i}\right)$, assume that $a\left(x_{l}\right)=a_{l}$ for each $i \in \mathscr{Z}$, in this case, we say that a interpolates $\left(a_{i}\right)$, or, equivalently, that $\left(a_{l}\right)$ is the discretization of the function $a$

Interpolation can be described geometrically, in terms of a projection More precisely, let $\Pi^{*}$ be the map sending the function $a$ of $x$ into the sequence $a_{i}=$ $a\left(x_{i}\right), \Pi^{*}$ is a surjective application between the phase spaces of the interpolated and the discrete systems The vector fields $X$, of Subsect 22 and their continuous 
homologues $X_{s}^{\prime}$ are related by

$$
X_{\varsigma}\left(\left(a_{l}\right)\right)=\left(T_{a} \Pi^{i}\right)\left(X_{\varsigma}^{\xi}(a)\right),
$$

where $T \Pi^{8}$ denotes the tangent map and $a$ is any function such that $\Pi^{8}(a)=\left(a_{l}\right)$ The geometrical property expressed by Eq (3 6) corresponds to the general notion of projectability for vector fields

\section{The Zero Spacing Limit and KdV Theory}

41 Field rescaling Our purpose in this section is to recover $\mathrm{KdV}$ theory from the interpolated KM system through the $s \mapsto 0$ limit As a preliminary step, we introduce (for $\varepsilon$ finite and arbitrary) a rescaled field variable $u$, related to $a$ by Eq ( 13 ) For any fixed $\varepsilon \neq 0$, we can describe the rescaling by saying that there is a diffeomorphism

$$
\Phi^{:} \quad \mathscr{A} \rightarrow \boldsymbol{U}, \quad a \mapsto u=\frac{a-2}{\varepsilon^{2}}
$$

(with $1 /$ an appropriate set of functions ${ }^{5}$ ) At any point $a \in \mathscr{A}$, the tangent map of $\Phi^{.}$and its adjoint are

$$
\begin{gathered}
T_{a} \Phi^{\varepsilon} \quad T_{a} \mathscr{A} \rightarrow T_{u} \|, \quad \dot{a} \mapsto \dot{u}=\frac{\dot{a}}{\varepsilon^{2}}, \\
T_{a}^{*} \Phi^{\varepsilon} \quad T_{u}^{*} \mathscr{U} \rightarrow T_{a}^{*} \mathscr{A}, \quad \delta u \mapsto \delta a=\frac{1}{\varepsilon^{2}} \delta u
\end{gathered}
$$

We can employ the diffeomorphism $\Phi^{\varepsilon}$ to transport on $\mathbb{U}$ the whole structure of the KM system The correspondents of the objects considered in the previous section will be denoted for simplicity with the same letters So, $\mathbb{U}$ carries a hierarchy of vector fields

$$
X_{s}^{i}(u)=\left.\left(T_{a} \Phi^{i}\right)\left(X_{s}^{i}(a)\right)\right|_{a=\left(\Phi^{\prime}\right)^{-1}(u)}=\left.\frac{1}{\varepsilon^{2}} X_{s}^{i}(a)\right|_{a=2+\xi^{2} u}
$$

and Hamiltonians

$$
f_{s}^{\varepsilon}(u)=\left.f_{s}^{\varepsilon}(a)\right|_{a=\left(\Phi^{\prime}\right)^{-1}(u)}=\left.f_{s}^{\varepsilon}(a)\right|_{a=2+\varepsilon^{2} u} .
$$

The first Poisson tensor on $\mathbb{M}$ is

$$
Q_{u}^{\varepsilon}=\left.T_{a} \Phi^{i} \circ Q_{a}^{\xi} \circ T_{a}^{*} \Phi^{i}\right|_{a=\left(\Phi^{*}\right)^{-1}(u)}=\left.\frac{1}{\varepsilon^{4}} Q_{a}^{\vdots}\right|_{a=2+\varepsilon^{2} u},
$$

i e, from $\mathrm{Eq}$ (2 2), $Q_{u}^{\varepsilon}=\frac{1}{2 \varepsilon^{4}}\left(\left(2+\varepsilon^{2} u\right) \Delta_{\varepsilon}\left(2+\varepsilon^{2} u\right)-\left(2+\varepsilon^{2} u\right) \Delta_{-\varepsilon}\left(2+\varepsilon^{2} u\right)\right)$ The second Poisson tensor $P_{u}^{\varepsilon}$ is constructed similarly

The Lax operator for the KM hierarchy on 01 is

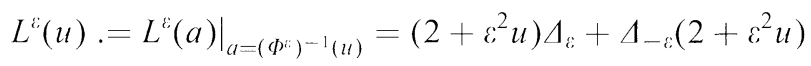

\footnotetext{
${ }^{5}$ If $x$ langes over the line $\mathbb{R}$, it is convenient to assume that, for all functions $a \in \mathscr{d}$ and $u \in \mathbb{\|}$, $a-2$ and $u$ be rapidly vanishing at infinity
} 
42 The $\varepsilon \mapsto 0$ limit The Taylor formula $\psi(x+r \varepsilon)=\sum_{J=0}^{+\infty}(1 / j !)\left(r^{\prime}\right)^{j} \frac{d^{\prime} \psi}{d d^{\prime}}(x)$, for the shift of a function $\psi$, means that $\Delta_{\ell:}=\exp \left(r \varepsilon \hat{c}_{1}\right)$ So, if we take the Lax operator $L^{\prime}(u)$ in Eq (47) and expand it in powers of $\varepsilon$ up to order 2, we find (similarly to $[$ Kup])

$$
L^{\varepsilon}(u)=4+2 \varepsilon^{2}\left(\partial_{r}+u\right)+O\left(\varepsilon^{3}\right)
$$

The appearence of the KdV Lax operator is a hint that we are following the right path towards our goal Now, let us pass to the vector fields $X_{s}^{\xi}$. The first one is given by

$$
X_{1}^{\prime}(u)=\frac{1}{\varepsilon^{2}}\left(2+\varepsilon^{2} u\right)\left[\left(2+\varepsilon^{2} u_{(\varepsilon)}\right)^{2}-\left(2+\varepsilon^{2} u_{(-\varepsilon)}\right)^{2}\right] .
$$

From the Taylor expansion of $u(x \pm \varepsilon)$, we have $u_{( \pm \varepsilon)}=u \pm \varepsilon u_{\curlyvee}+\frac{1}{2} \varepsilon^{2} u_{u}++$ $\left(\varepsilon^{6} / 720\right) u_{\text {rrrrr }}+O\left(\varepsilon^{7}\right)$, which implies

$$
\begin{aligned}
& X_{1}(u)=16 c u_{1}+\frac{8}{3} c^{3}\left(u_{1}+6 u u_{\imath}\right)
\end{aligned}
$$

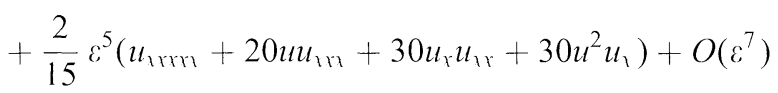

Similarly, we find

$$
\begin{aligned}
& X_{2}^{\prime}(u)=384 \varepsilon u_{1}+128 \varepsilon^{3}\left(u_{r r}+6 u u_{\imath}\right) \\
& +\frac{96}{5} \varepsilon^{5}\left(u_{\imath \mathrm{rr}}+\frac{40}{3} u u_{\imath \mathrm{r}}+\frac{70}{3} u_{\curlyvee} u_{\Upsilon \imath}+30 u^{2} u_{\imath}\right)+O\left(\varepsilon^{7}\right), \\
& X_{3}^{\varkappa}(u)=7680 \varepsilon u_{x}+3840 c^{3}\left(u_{1 x}+6 u u_{x}\right) \\
& +960 \varepsilon^{5}\left(u_{\imath \imath \eta}+12 u u_{\imath n}+22 u_{\gamma} u_{\imath x}+30 u^{2} u_{\gamma}\right)+O\left(\varepsilon^{7}\right)
\end{aligned}
$$

The inspection of the above expansions suggests that the first three vector fields of the $\mathrm{KdV}$ hierarchy could be recovered in the $\varepsilon \mapsto 0$ limit by suitable recombinations, so that the lowest order term in the expansion of each recombined field is a $\mathrm{KdV}$ vector field. Indeed, this is obtained introducing, for each finite $\varepsilon$, the vector fields

$$
Z_{1}^{\varepsilon}=X_{1}^{\varepsilon}, \quad Z_{2}^{i}=X_{2}^{i}-24 X_{1}^{\varepsilon}, \quad Z_{3}^{\varepsilon}=X_{3}^{\varepsilon}-40 X_{2}^{\delta}+480 X_{1}^{\varepsilon}
$$

(that we can consider to be defined on both phase spaces $\mathscr{A}$ and $\mathscr{U}$ ) The choice of the coefficients in Eq (4 13) implies that

$$
\begin{aligned}
Z_{1}^{i}(u) & =16 \varepsilon u_{x}+O\left(\varepsilon^{3}\right)=16 \varepsilon_{1}^{\mathrm{KdV}}(u)+O\left(\varepsilon^{3}\right), \\
Z_{2}^{i}(u) & =64 \varepsilon^{3}\left(u_{u}+6 u u_{x}\right)+O\left(\varepsilon^{5}\right)=64 \varepsilon^{3} Z_{2}^{\mathrm{KdV}}(u)+O\left(\varepsilon^{5}\right), \\
Z_{3}^{\varepsilon}(u) & =256 \varepsilon^{5}\left(u_{\mathrm{rrrr}}+10 u u_{u}+20 u_{\uparrow} u_{\curlywedge}+30 u^{2} u_{x}\right)+O\left(\varepsilon^{7}\right) \\
& =256 \varepsilon^{5} Z_{3}^{\mathrm{KdV}}(u)+O\left(\varepsilon^{7}\right)
\end{aligned}
$$


These experimental facts lead us to conjecture that we could generate all vector fields of the $\mathrm{KdV}$ hierarchy with the same recombination method This result will be presented in the next section, for the moment, we maintain an experimental attitude and test the $\varepsilon \mapsto 0$ behaviour of the first KM Hamiltonians We have

$$
\begin{aligned}
f_{0}^{\prime \prime}(u) & =\int d x \log \left(2+\varepsilon^{2} u\right) \\
& =\log 2 \int d x+\frac{\varepsilon^{2}}{2} \int d x u-\frac{\varepsilon^{4}}{8} \int d x u^{2}+\frac{\varepsilon^{6}}{24} \int d x u^{3}+O\left(\varepsilon^{8}\right), \\
f_{1}^{\prime}(u) & =\int d x\left(2+\varepsilon^{2} u\right)^{2}=4 \int d x+4 \varepsilon^{2} \int d x u+\varepsilon^{4} \int d x u^{2}, \\
f_{2}^{\sharp}(u) & =\int d x\left(\frac{1}{2}\left(2+\varepsilon^{2} u\right)^{4}+\left(2+\varepsilon^{2} u(:)\right)^{2}\left(2+\varepsilon^{2} u\right)^{2}\right) \\
& =24 \int d x+48 \varepsilon^{2} \int d x u+36 \varepsilon^{4} \int d x u^{2}+4 \varepsilon^{6} \int d x\left(3 u^{3}-2 u_{1}^{2}\right)+O\left(\varepsilon^{8}\right)
\end{aligned}
$$

So, setting

$$
\begin{aligned}
& h_{0}^{i}=f_{0}^{i}-\log 2 \int d x, \quad h_{1}^{i}=f_{1}^{\varepsilon}-8 f_{0}^{\varepsilon}-(4-8 \log 2) \int d x, \\
& h_{2}^{*}=f_{2}^{\varepsilon}-24 f_{1}^{\varepsilon}+96 f_{0}^{\varepsilon}-(96 \log 2-72) \int d x \text {, }
\end{aligned}
$$

we infer

$$
\begin{aligned}
& h_{0}^{i}(u)=\frac{1}{2} \varepsilon^{2} \int d x u+O\left(\varepsilon^{4}\right)=\varepsilon^{2} h_{0}^{\mathrm{KdV}}(u)+O\left(\varepsilon^{4}\right), \\
& h_{1}^{\varepsilon}(u)=2 \varepsilon^{4} \int d x u^{2}+O\left(\varepsilon^{6}\right)=4 \varepsilon^{4} h_{1}^{\mathrm{KdV}}(u)+O\left(\varepsilon^{6}\right), \\
& h_{2}^{\varepsilon}(u)=8 \varepsilon^{6} \int d x\left(2 u^{3}-u_{r}^{2}\right)+O\left(\varepsilon^{8}\right)=16 \varepsilon^{6} h_{2}^{\mathrm{KdV}}(u)+O\left(\varepsilon^{8}\right)
\end{aligned}
$$

Finally, let us consider the Poisson tensors $Q^{\varepsilon}, P^{\&}$ (see Eq (46)) We have

$$
\begin{aligned}
& Q_{u}^{\varepsilon}(\delta u)=\frac{4}{\varepsilon^{3}} \delta u_{x}+\frac{2}{3 \varepsilon}\left(\delta u_{\uparrow}+6 u \delta u_{\curlywedge}+3 u_{x} \delta u\right)+O(\varepsilon), \\
& P_{u}^{\xi}(\delta u)=\frac{64}{\varepsilon^{3}} \delta u_{\Upsilon}+\frac{80}{3 \varepsilon}\left(\delta u_{r x x}+\frac{24}{5} u \delta u_{\Upsilon}+\frac{12}{5} u_{\Upsilon} \delta u\right)+O(\varepsilon),
\end{aligned}
$$

this implies

$$
Q_{u}^{\varepsilon}=\frac{4}{\varepsilon^{3}} \hat{\partial}_{r}+O\left(\frac{1}{\varepsilon}\right), \quad S_{u}^{\varepsilon}=\frac{16}{\varepsilon}\left(\hat{c}_{\mathrm{rx}}+4 u \hat{\partial}_{\curlyvee}+2 u_{\Upsilon}\right)+O(\varepsilon),
$$

\footnotetext{
${ }^{6}$ In the penodic case, $\int d x=1$ (the total measune of the torus $\pi$ ) We recall that we have defined $\int d:=0$ if 1 langes over the line $\mathbb{R}$ : see the footnote in Sect 3 In computing the expansions of the Hamiltonians, we take into account that, for any polynomial $\varphi$ in $u$ and its derivatives, it is $\int d \wedge \varphi_{1}=0$; this is granted when $x$ tanges over $\mathbb{T}$, or, alternatively, when $\downarrow$ ranges over $\mathbb{R}$ and $u$ is rapidly vanishing at infinity
} 
where we have put

$$
S^{\varepsilon}=P^{\varepsilon}-16 Q^{\varepsilon}
$$

In $\mathrm{Eq} \mathrm{(420),} \mathrm{one} \mathrm{recognizes} \mathrm{the} \mathrm{KdV}$ Poisson tensors, this fact will be basic in the next section We observe that, for any finite $\varepsilon, Q^{\varepsilon}$ and $S^{*}$ form a pair of compatible Poisson tensors on the phase space II Also, the definition (421) of $S^{\prime:}$ is consistent with the recombination rules found for the first vector fields and Hamiltonian functions, in the sense that we still have a recursion scheme, in fact, using Eqs (4 13), (4 16), (4 21), and Eq (3 1), one can check that, for the lowest values of $s$,

$$
Z_{s+1}^{i}=Q^{\varepsilon} d h_{s+1}^{i}=S^{\varepsilon} d h_{s}^{i}
$$

We are now going to develop systematically the approach suggested here, so as to recover not only the first vector fields and Hamiltonians, but the whole $\mathrm{KdV}$ hierarchy

Remark. From our viewpoint, the role of the first $\mathrm{KM}$ vector field $X_{1}^{:}$is simply to give, in the $\varepsilon \vdash 0$ limit, the vector field $Z_{1}^{\mathrm{KdV}}(u)=u_{1}$, i.e, the infinitesimal generator of space translations on $\mathbb{M}$, to obtain the ordinary $\mathrm{KdV}$ equation (1 1), i e, the vector field $Z_{2}^{\mathrm{KdV}}$, we take the limit of the combination $X_{2}^{\varepsilon}-24 X_{1}^{*}$ For completeness, we compare this construction with Kupershmidt's derivation of $\mathrm{Eq}$ ( 111 ) Kupershmidt employs only the first $\mathrm{KM}$ vector field $X_{1}^{\xi}$, according to Eq (4 10), the evolution equation $d u / d t_{1}=X_{1}^{z}(u)$ becomes, at the lowest orders in $c$,

$$
u_{\iota_{1}}=16 \varepsilon u_{\imath}+\frac{8}{3} \varepsilon^{3}\left(u_{u}+6 u u_{\imath}\right) .
$$

The manipulation performed by Kupershmidt on this equation is described in terms of his abstract language of evolutionary derivations, but amounts essentially to consider a space-time coordinate change

$$
\xi=x+16 \varepsilon t_{1}, \quad \tau_{1}=\frac{8}{3} c^{3} t_{1}
$$

under which Eq (423) becomes $u_{\tau_{1}}=u_{\xi \xi}+6 u u_{\xi}$ The $\tau_{1}$ variable is clearly a rescaled time, while $\xi$ can be interpreted as the space coordinate for an observer translating with speed $16 \%$ with respect to the lattice ${ }^{7}$, the introduction of this observer, which is a standard trick in the literature, is essentially motivated by the aim to reabsorb the term in $c u_{\text {}}$ from $\mathrm{Eq}$ (423) No attempt is made in [Kup] to recover the subsequent vector fields of the $\mathrm{KdV}$ hierarchy

When we subtract from $X_{2}^{8}$ the vector field $X_{1}^{i}$ to get the $\mathrm{KdV}$, we essentially perform the transformation (4 24) on the evolution equation $d u / d t_{2}=X_{2}(u)$, because $X_{1}(u)$ behaves for $\varepsilon \mapsto 0$ as $16 \varepsilon u_{1}$, which is the generator of the one-parameter group of transformations $u(x) \mapsto u\left(x+16 \varepsilon t_{1}\right)$ No interpretation of this sort, in terms of a space-time variable change, is possible for the higher order recombinations such as the vector field $X_{3}^{i}-40 X_{2}^{i}+480 X_{1}^{i}$

\footnotetext{
${ }^{7}$ One should not be astonished that $:$ can be both a lattice spacing and a speed, since all the space-time and field variables employed in the paper are dimensionless
} 


\section{The General Recombination Scheme}

The general recombination formulas for the infinitely many vector fields $X_{;}$and Hamiltonians $f_{s}$ will be given in terms of a set of coefficients $\left(c_{s,}\right)$

5.1. Definition. $\left.\left(c_{s, j}\right)_{1,0}\right)_{2}$, is the family of real coefficients defined recursitely in the following way

$$
c_{s+1,1}= \begin{cases}-c_{00}=1, & \\
c_{s,-1}-16 c_{s,}\left(\begin{array}{c}
s+2 \\
s+1
\end{array}\right)-\sum_{l=1}^{s} 4^{\prime}\left(\begin{array}{c}
2 i \\
l
\end{array}\right)\left(c_{s,-1}-16 c_{s l}\right) & \text { for } j=0 \\
1 & \text { for } j=1,2, \quad, s \\
& \text { for } j=s+1\end{cases}
$$

(here $\left(\begin{array}{l}1 \\ h\end{array}\right)$ denotes as usual the binomial coefficient, for each pair $r, k$ )

One can arrange the above coefficients into an infinite lower triangular matrix

$$
\left(\begin{array}{ccccc}
c_{00} & 0 & 0 & 0 & \\
c_{10} & c_{11} & 0 & 0 & \ldots \\
c_{20} & c_{21} & c_{22} & 0 &
\end{array}\right)
$$

Knowledge of any row in this matrix allows to compute the subsequent one, according to Eq (52), it should be noted that all elements $c_{s s}$ on the main diagonal are equal to 1 , and that

$$
c_{s 0}=-4^{s}\left(\begin{array}{c}
2 s \\
s
\end{array}\right)-\sum_{i=1}^{s-1} 4^{\prime}\left(\begin{array}{c}
2 i \\
i
\end{array}\right) c_{s i} \quad(s=1,2,3, \quad)
$$

5.2. Definition. For each \&, $Z_{s} \quad(s=1,2,3$, $)$ and $h_{s} \quad(s=0,1,2, \quad)$ are the vector fields and the Hamiltonians defined by

$$
\begin{gathered}
Z_{\zeta}^{i}=\sum_{j=1}^{s} c_{s j} X_{j}^{i}, \\
h_{\varsigma}^{i}=\sum_{j=0}^{s} c_{s j} f_{j}^{i}-c_{s},
\end{gathered}
$$

where the constants $c$, are given by

$$
c_{s}=\left(c_{s 0} \log 2+\sum_{j=1}^{s} \frac{2^{2 \jmath-1}}{j}\left(\begin{array}{c}
2 j \\
j
\end{array}\right) c_{s,}\right) \int d x .
$$

For the lowest values of $s$, Eqs $(51-7)$ give the vector fields and the Hamiltonians considered in the previous section

5.3. Proposition. Let $Q^{\varepsilon}$ and $S^{\circ}=P^{i}-16 Q^{i}$ be the Poisson tensors considered in Sect 4 Then $Q^{\prime} d h_{0}^{*}=0$ and

$$
Z_{s+1}^{i}=Q^{i} d h_{s+1}^{i}=S^{i} d h_{\uparrow}^{i} \quad(s=0,1,2, \quad)
$$


Proof We already know that $h_{0}^{\varepsilon}=f_{0}^{\varepsilon}-c_{0}$ is a Casimir of $Q^{\varepsilon}$ From Eqs (5 6) (3 1$)$ and $(55)$ it follows that

$$
Q^{\varepsilon} d h_{\varsigma+1}^{\varepsilon}=\sum_{j=0}^{s+1} c_{s+1, j} Q^{\varepsilon} d f_{j}^{\varepsilon}=\sum_{j=1}^{s+1} c_{s+1, j} X_{j}^{\varepsilon}=Z_{s^{2}+1}^{\varepsilon}
$$

Also taking into account the definition of $S^{\prime \prime}$ and the recursion rule (52), we obtain

$$
S^{i} d h_{\mathrm{s}}^{\varepsilon}=\sum_{j=0}^{s} c_{s /}\left(P^{i}-16 Q^{\varepsilon}\right) d f_{j}^{i}=X_{s+1}^{\varepsilon}+\sum_{j=1}^{\varsigma}\left(c_{s, j-1}-16 c_{\varsigma,}\right) X_{j}^{i}=Z_{s+1}^{i}
$$

It should be noted that our argument entails only a part of the recurrence law, namely the formula for $c_{s+1, j}$ for $j$ running from 1 to $s+1$; for the moment, the rule for $c_{i+1,0}$ is immaterial, as well as the explicit expression stipulated for the constants cs in Eq (57). On the contrary, these prescriptions will be essential in the sequel, to characterize the behaviour of the Hamiltonians $h_{s}$ at the lowest orders in $\varepsilon$

For convenience, we now restate a result essentially known from the previous section

5.4. Proposition. We have

$$
Q_{u}^{\varepsilon}=\frac{4}{\varepsilon^{3}} Q_{u}^{\mathrm{KdV}}+O\left(\frac{1}{\varepsilon}\right), \quad S_{u}^{u}=\frac{16}{\varepsilon} S_{u}^{\mathrm{KdV}}+O(\varepsilon)
$$

These are the leading terms of two expansions of the form

$$
Q_{u}^{\varepsilon}=\sum_{m=0}^{+\infty} \varepsilon^{2 m-3} Q_{2 m-3, u}, \quad S_{u}=\sum_{m=0}^{+\infty} \varepsilon^{2 m-1} S_{2 m-1, u}
$$

Proof The method of Sect 4 ensures that such expansions in $\varepsilon$ are possible, that the leading terms are in $1 / \varepsilon^{3}$ for $Q_{u}$ and $1 / \varepsilon$ for $S_{u}^{\varepsilon}$ and have explicit expressions as in $\mathrm{Eq}$ (5 11) (compare with Eq. (4 20)). It remains to show that, in both cases, only odd powers of $\varepsilon$ appear in the developments. Indeed, it is evident from Eqs (4 6), (2 2) and (2 3) that $Q_{u}^{z}=-Q_{u}^{-\varepsilon}$ and $P_{u}^{z}=-P_{u}^{-\varepsilon}$, the tensor $S_{u}^{\varepsilon}$ is also an odd function of $\varepsilon$, since it is a linear combination of $Q_{u}$ and $P_{u}^{\varepsilon}$

From the previous two propositions, we finally infer

5.5. Proposition. One has

$$
\begin{gathered}
h_{\varsigma}^{\xi}(u)=4^{\varsigma} \varepsilon^{2 s+2} h_{\varsigma}^{\mathrm{KdV}}(u)+O\left(\varepsilon^{2 \varsigma+4}\right) \quad(s=0,1,2, \quad), \\
Z_{\varsigma}^{\varepsilon}(u)=4^{s+1} c^{2 \varsigma-1} Z_{\varsigma}^{\mathrm{KdV}}(u)+O\left(\varepsilon^{2 s+1}\right) \quad(s=1,2,3, \quad)
\end{gathered}
$$

Proof The essential point consists in showing Eq. (5 13), our arguments will be divided in three steps

Step 1 We start by analyzing the expansions in $\varepsilon$ of the Hamiltonians $f_{s}$

Let us employ the representation $f_{s}^{\prime}(u)=\left.\frac{1}{2 s} \operatorname{Tr} L_{i s}^{2 s}(a)\right|_{a=2+\varepsilon^{2} u}$, holding for $s=$

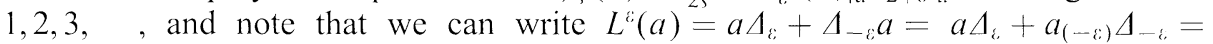


$\sum_{l= \pm 1} a_{\left(\frac{1}{2} /:-\frac{1}{2} i\right)} \Delta_{l}$ This implies

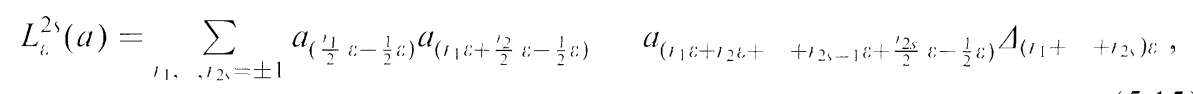

so, taking the trace, we find

$$
\begin{aligned}
& f_{5}^{\prime \prime}(u)
\end{aligned}
$$

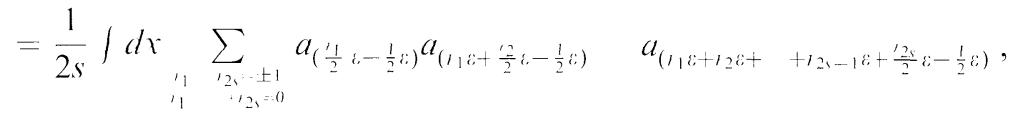

with $a=2+\varepsilon^{2} u$ We wish to make explicit the lowest order terms in the expansion in $\varepsilon$ of $(516)$ To this purpose we note that, for each $r_{1}, \ldots, r_{k}$, it is

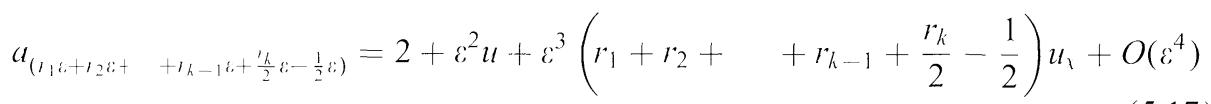

So, for each $r_{1}, \quad, r_{2}$, we have

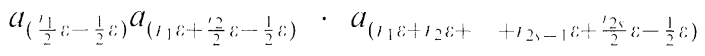

$$
\begin{aligned}
& =2^{2 s}+2^{2 s} s \varepsilon^{2} u+2^{2 s-1} \varepsilon^{3}\left(\sum_{k=1}^{2 s}\left(2 s-k+\frac{1}{2}\right) r_{k}-s\right) u_{r}+O\left(\varepsilon^{4}\right)
\end{aligned}
$$

If we integrate over $x$, the term in $\varepsilon^{3}$ in the above formula disappears, because $\int d r u_{1}=0$, if we also sum over $r_{1}, ., r_{2 s}$, we conclude that

$$
f_{s}^{\prime \prime}(u)=2^{2 s-1} \#(s)\left(\frac{1}{s} \int d x+\varepsilon^{2} \int d x u\right)+O\left(\varepsilon^{4}\right),
$$

where $\#(s)$ is the number of finite sequences $\left(r_{1}, ., r_{2 s}\right)$ with values in \pm 1 and zero sum Elementary combinatorics gives $\#(s)=\left(\begin{array}{c}2 s \\ s\end{array}\right)$

We note that developing $f_{s}(u)$ up to $O\left(\varepsilon^{4}\right)$, we find no terms of order $\varepsilon$ and $\varepsilon^{3}$ In general, no odd powers of $\varepsilon$ appear in the development this follows from the identity $f_{s}^{-c}(u)=f_{s}^{\prime \prime}(u)$, which can be derived directly, without expanding, from the representation $(516)$ So, we can state that

$$
f_{s}^{\prime \prime}(u)=\sum_{l=0}^{+\infty} \varepsilon^{2 l} f_{s, 2 l}(u)
$$

in particular, the first two terms are as we found above, i e,

$$
f_{s 0}(u)=\frac{2^{2 s-1}}{s}\left(\begin{array}{c}
2 s \\
s
\end{array}\right) \int d x, \quad f_{s 2}(u)=2^{2 s-1}\left(\begin{array}{c}
2 s \\
s
\end{array}\right) \int d x u
$$

The above equations hold for each $s \geqq 1$ For $s=0$, the corresponding Hamiltonian $f_{0}^{:}$can also be expanded as in Eq $(520)$, the first two terms being

$$
f_{00}(u)=\log 2 \int d x, \quad f_{02}(u)=\frac{1}{2} \int d x u
$$


For our purposes, it is not necessary to make explicit the terms of order $\geqq 4$ in the expansions of the Hamiltonians, it suffices to remark that, for $2 l \geqq 4$, each functional $f_{s, 2 l}(u)$ is (the integral of) a polynomial in $u$ and its derivatives, containing no costant term and no linear term in $u$

Step 2 Let us come to the Hamiltonians $h_{s}^{:}(s=0,1,2$, .) Each of them has an expansion

$$
h_{s}(u)=\sum_{l=0}^{+\infty} c^{2 l} h_{\varsigma, 2 l}(u)
$$

Our previous considerations enable us to state that

$$
h_{00}(u)=0, \quad h_{\varsigma 0}(u)=h_{\varsigma 2}(u)=0 \quad(s=1,2,3, .)
$$

This follows immediately from the definition (56) of $h$ as a linear combination of the Hamiltonians $f_{j}$, from Eqs (5 21), (5 22) and from Eqs (5 4), (5 7) Now, we state that it is also

$$
h_{\triangleleft 4}(u)=h_{{ }^{6} 6}(u)=\quad=h_{\triangleleft, 2 \varsigma}(u)=0
$$

for each $s \geqq 2$ If $s=2$, this is already known (see Eq (4 17)) The general proof can be obtained by induction so, we assume that Eq ( 525$)$ holds for a given $s$ and show that it also holds for $s+1$, on the grounds of the biHamiltonian recursion relations In fact, we have $Q_{u l}^{i} d_{u} h_{\varsigma+1}^{\dagger}=S_{u}^{i} d_{u} h_{\varsigma}^{\zeta}=O(1 / \varepsilon) O\left(\varepsilon^{\dagger+2}\right)$, i e ,

$$
Q_{l t}^{i} d_{u} h_{s+1}^{*}=O\left(\varepsilon^{s+1}\right)
$$

The expansion of $Q_{u l}^{i} d_{u} h_{s+1}^{z}$ can be computed in terms of the developments (5 12) for $Q_{u}^{*}$ and $(523-24)$ for $h_{s+1}^{*}$ If we set to zero the terms in $\varepsilon^{k}$ for $k \leqq s$, so as to satisfy Eq (5 26), we obtain a triangular system of $s-1$ equations

$$
\begin{aligned}
& Q_{-3{ }_{u}} d_{u} h_{5+14}=0, \quad Q_{-3 . u} d_{u} h_{\uparrow+1.6}+Q_{-1 . u} d_{u} h_{5+14}=0 \text {, }
\end{aligned}
$$

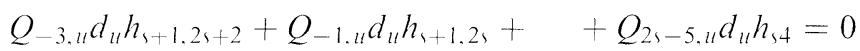

Let us analyze this system, recalling that $Q_{-3, u}=4 \hat{c}_{\text {, }}$ and that the functionals $h_{\uparrow+12} /$ are integrals of polynomial densities in $u$ and its derivatives, containing neither constants nor linear terms in $u$ Due to these features, the first equation $\left(d_{l l} h_{s+14}\right)_{1}=0$ in the system (527) implies $h_{s+1.4}=0$, substituting in the second equation we find that $\left(d_{11} h_{5+16}\right)_{1}=0$, and so $h_{s+16}=0$, iterating this argument, we infer $d_{u l} h_{\checkmark, 1,2 l}=0$ for each $l \leqq s+1$

In conclusion, we have proved $\mathrm{Eq}$ ( 525 ), which implies, together with $\mathrm{Eq}$ (5 24),

$$
h_{s}^{\sharp}(u)=\varepsilon^{2 s+2} h_{s} s+2(u)+O\left(\varepsilon^{2 s+4}\right) \quad(s=0,1,2, \quad) .
$$

Step 3 We substitute Eq ( 528 ) in the biHamiltonian recursion formula ( 58 ), using again Eqs (5 11-12), we obtain that the sequence of functionals $h_{5,2+2}$ satisfies the recursion scheme

$$
Q_{u}^{\mathrm{KdV}} d_{u} h_{s+1,2 s+4}=4 S_{u}^{\mathrm{KdV}} d_{u} h_{\uparrow, 2 \uparrow+2} \quad(s=0,1,2, .),
$$

which is very similar to Eq (2 23) The basic features of the functionals $h_{s, 2 s+2}$ with $s \geqq 1$ (differential polynomials in $u$, with no constants and no linear terms in $u$ ) have 
been already emphasized, on the other hand, the recursion scheme (2 23) admits no more than one solution with such features, for each choice of the starting point. The starting point of the sequence $h_{\varsigma, 2 \varsigma+2}$ is $h_{02}(u)=\frac{1}{2} \int d x u=h_{0}^{\mathrm{KdV}}(u)$, from here, and from Eq (2 23), one immediately gets

$$
h_{\varsigma, 2 \uparrow+2}(u)=4^{\varsigma} h_{\varsigma}^{\mathrm{KdV}}(u)
$$

So, Eq $(513)$ is proved.

The remaining Eq (5 14) follows straightforwardly, expanding to the lowest order in $\varepsilon$ the identity $Z_{\varsigma}=Q_{s} d h_{\varsigma}$

The connection between the KM system and $\mathrm{KdV}$ theory can be rephrased in terms of the Lax formalism By definition, the $Z_{s}$ vector fields are linear combinations of the $X_{s}^{\prime}$ 's, which admit the Lax formulations (34), so, if $d / d 0$, denotes the derivative along $Z_{s}$, we have

$$
\frac{d L^{\xi}}{d \theta_{s}}=\left[B_{s}^{i}, L^{i}\right], \quad B_{\varsigma}^{i}(u)=\sum_{j=1}^{s} c_{\zeta,} A_{j}^{i}(u)
$$

We already know the lower terms in development of $L^{\prime}(u)$, we complete our analysis considering the behaviour of $B_{s}(u)$ and summarize the results in the following

5.6. Proposition. One has

$$
\begin{gathered}
L^{\varepsilon}(u)-4=2 \varepsilon^{2} L^{\mathrm{KdV}}(u)+O\left(\varepsilon^{3}\right), \\
B_{s}^{i}(u)=4^{s+1} \varepsilon^{2 s-1} B_{s}^{\mathrm{KdV}}(u)+O\left(\varepsilon^{2 s}\right) \quad(s=1,2,3, \quad)
\end{gathered}
$$

Proof The result concerning the Lax operator is already known (see Eq. (4 8)) To derive Eq (533), we proceed in three steps, the integer $s$ is fixed throughout the proof

Step 1 For subsequent use, it is useful to emphasize a feature of the operator $B_{s}(u)$ at the point $u=0$. On account of $\mathrm{Eq}(47)$, we have $L^{i}(0)=2 \Delta_{i}+2 \Delta_{-i,}$, so, from the expression (34) of $A_{;}$and $\mathrm{Eq}(531)$ it follows that $B_{5}(0)$ is a linear combination with constant coefficients of differences of the form $\Delta_{l \%}-\Delta_{-r}$, with $r$ an integer between 0 and $2 s$ Expanding in $\varepsilon$, we find only odd powers of $\varepsilon$ and $\hat{c}_{1}$

$$
B_{\varsigma}^{c}(0)=\sum_{m=0}^{+\infty} b_{s} 2 m+1 \varepsilon^{2 m+1} \hat{c}_{\imath}^{2 m+1}
$$

where $b_{\uparrow 1}, b_{\varsigma 3}$, are real constants whose explicit expression is not relevant for our purposes At a generic point $u$, we have the expansion

$$
B_{\varsigma}^{\varepsilon}(u)=\sum_{p=0}^{+\infty} \varepsilon^{p} B_{\varsigma p}(u)
$$

for each $p, B_{s p}(u)$ is a differential operator in $\hat{c}_{1}$, whose coefficients are polynomials in $u$ and its derivatives In particular, at the point $u=0$ we find, on account of Eq (5 34),

$$
B_{\varsigma p}(0)=0 \quad \text { for } p \text { even, } \quad B_{\varsigma p}(0)=b_{\varsigma p} \hat{c}_{\imath}^{p} \quad \text { for } p \text { odd }
$$


Step 2 The $\varepsilon \mapsto 0$ limits of the $Z_{s}$ vector fields are the $\mathrm{KdV}$ vector fields $Z_{\mathrm{s}}^{\mathrm{KdV}}$, according to Eq (5 14), so, by comparing the Lax formulations (5 31) and (2.25) we infer

$$
\left[B_{s}^{\prime \prime}(u), L^{i}(u)\right]=2^{2 s+3} \varepsilon^{2 s+1}\left[B_{\varsigma}^{\mathrm{KdV}}(u), L^{\mathrm{KdV}}(u)\right]+O\left(\varepsilon^{2 s+2}\right)
$$

Our aim is to obtain from this equation the maximum amount of information about the operator $B_{s}$ To this purpose, we use the expansion (5 35), and write $L^{\varepsilon}(u)=4+$ $2 \varepsilon^{2} L^{\mathrm{KdV}}(u)+\sum_{q=3}^{+\infty} \varepsilon^{q} L_{q}(u)$, for each $q, L_{q}(u)$ is a differential operator Substituting these developments in Eq (5.37), and considering the terms up to $\varepsilon^{2 s}$, we obtain a triangular system of $2 s-1$ equations

$$
\begin{gathered}
{\left[B_{s 0}(u), L^{\mathrm{KdV}}(u)\right]=0, \quad 2\left[B_{\varsigma 1}(u), L^{\mathrm{KdV}}(u)\right]+\left[B_{s 0}(u), L_{3}(u)\right]=0, \quad,} \\
2\left[B_{s, 2 \varsigma-2}(u), L^{\mathrm{KdV}}(u)\right]+\sum_{u=3}^{2 s}\left[B_{s, 2 s-q}(u), L_{q}(u)\right]=0
\end{gathered}
$$

Furthermore, extracting from $(5.37)$ the terms in $\varepsilon^{2 s+1}$, we find

$2\left[B_{s, 2 s-1}(u), L^{\mathrm{KdV}}(u)\right]+\sum_{q=3}^{2 s+1}\left[B_{s, 2 s+1-q}(u), L_{q}(u)\right]=2^{2 \varsigma+3}\left[B_{s}^{\mathrm{KdV}}(u), L^{\mathrm{KdV}}(u)\right]$

Step 3 By discussing the triangular system (5 38), we will show that $B_{s p}(u)=0$ for $p=0,1,2, \quad, 2 s-2$, substituting this result in $\mathrm{Eq}$ (5 39), we will be able to determine the operator $B_{s, 2 s-1}(u)$, and this will conclude the proof

Let us start from the first equation in the system (5 38), which asserts that the differential operator $B_{s 0}(u)$ is in the centralizer of $L^{\mathrm{KdV}}(u)$ The well known characterization of the centralizer (see for example [DS]) implies in this case that $B_{\varsigma_{0}}(u)$ is a linear combination of nonnegative integer powers of $L^{\mathrm{KdV}}(u)$

$$
B_{s 0}(u)=\sum_{j} b_{s 0 /}\left(L^{\mathrm{KdV}}\right)^{j}(u)
$$

where the $b_{s 0}$, are constant coefficients, independent of $u$ On the other hand, at the point $u=0$ the 1 h.s of Eq. (5 40) vanishes on account of Eq (536), while $\left(L^{\mathrm{KdV}}\right)^{\prime}(0)=\hat{c}_{1}^{2 \prime}$, so, we conclude that $b_{s 0}=0$ for each $j$, i e, $B_{s 0}(u)=0$ at each point $u$

Inserting this result in the second equation of the system (538), we obtain that also $B_{\triangleleft 1}(u)$ belongs to the centralizer of $L^{\mathrm{KdV}}(u)$, so,

$$
B_{\mathrm{s} 1}(u)=\sum_{j} b_{\varsigma_{1 j}}\left(L^{\mathrm{KdV}}\right)^{\prime}(u),
$$

where $j$ ranges over a set of nonnegative integers and the $b_{s 1 j}$ are real constants Again we fix our attention on the point $u=0$, here the $1 \mathrm{~h} \mathrm{~s}$ of Eq $(541)$ is $b_{51} \hat{\partial}_{1}$ due to Eq (536), while the rh.s is $\sum_{l} b_{s 1,} c_{r}^{2 l}$ By comparison, we conclude that the constants $b_{s 1}$ and $b_{s 1 j}$ all vanish, i e, that $B_{s 1}(u)=0$

The above arguments can be iterated on the remaining equations of the system (5 38), one finally obtains that, at each point $u$,

$$
B_{s p}(u)=0 \quad(p=0,1, \quad, 2 s-2)
$$




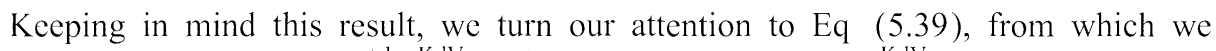
infer that $B_{5,2,-1}(u)-4^{s+1} B_{s}^{\mathrm{KdV}}(u)$ is in the centralizer of $L^{\mathrm{KdV}}(u)$. So,

$$
B_{\varsigma, 2 s-1}(u)=4^{s+1} B_{\varsigma}^{\mathrm{KdV}}(u)+\sum_{j} b_{s, 2 \varsigma-1, j}\left(L^{\mathrm{KdV}}\right)^{j}(u)
$$

To determine the constants $b_{\{.2,-1,1}$, we use again the trick of specializing Eq ( 543 ) to the point $u=0$, here the $1 \mathrm{~h} \mathrm{~s}$ is $b_{s, 2 s-1} \hat{c}_{\mathrm{s}}^{2 s-1}$ due to Eq (536), while the r h.s is $4^{2 s} \hat{c}_{r}^{2 s-1}+\sum_{j} b_{s, 2 s-1, j} \partial_{r}^{2 j}$ By comparison, we infer $b_{\varsigma, 2 s-1}=4^{2 s}$ and $b_{\uparrow, 2 \varsigma-1,1}=0$ for each $j$ In conclusion, at any point $u$ we have

$$
B_{\varsigma, 2 \varsigma-1}(u)=4^{s+1} B_{\varsigma}^{\mathrm{KdV}}(u) \text {; }
$$

the thesis (5 33 ) follows from this and from Eq (5 42)

Propositions 5 4, 55 and 56 contain the main results of the paper, of course, their statements can be written as in Eqs (1.6), (1.5) and (1 8 ) of the Introduction.

Let us note that Prop. 56 on the Lax pairs rests on the previous result in Prop 5 5, which has been proved using systematically the biHamiltonian recursion relations, so, in our approach, the continuous limit of the biHamiltonian scheme also gives the basic information about the limit of the Lax formalism

\section{Final Remarks}

In this paper, we have presented a zero-spacing limit procedure for the KM system, drawing attention to its algebraic/geometric structures (hierarchical organization of the conservation laws, biHamiltonian structure and Lax formalism) The convergence of the limit procedure for the solutions of the associated evolution equations deserve a separate discussion, it is outside our present purposes to give a systematic treatment of this topic, so we will limit ourselves to an example and some remarks

A first exercise for going inside this matter is the study of the limit process for the soliton solutions; here, we will analyze the one-soliton case for the first three vector fields of the hierarchy It will turn out that the recombination principle employed throughout this paper plays a basic role also in the present discussion the coefficients $c_{s}$, of Sects $4-5$ also control the recombination of the frequencies in the soliton solutions, and the prescriptions given for such coefficients allow to obtain the $\mathrm{KdV}$ one-soliton solutions through the limit process

Let us consider the discrete KM system, in the infinite case (the index $i$ labelling the sites runs over $\mathbb{Z}$ ) Let $X_{1}$ be the vector field given by Eq (2.7), it is known that the evolution equation $d a / d t_{1}=X_{1}(a)$ admits the one-soliton solution

$$
a_{l}\left(t_{1}\right)=2 \sqrt{\frac{\cosh (3 K)+\cosh 2\left[K(i-1 / 2)+v_{1}(K) t_{1}+A\right]}{\cosh (K)+\cosh 2\left[K(i-1 / 2)+v_{1}(K) t_{1}+A\right]}},
$$

depending on the arbitrary real parameters $K$ and $A$, with $v_{1}(K)=8 \sinh (2 K)$ Equation (6 1$)$ is equivalent to an equation appearing in [Man], clearly, the ratio $1_{1}(K) / K$ gives the speed of the soliton center, whose position at time $t_{1}=0$ is controlled by $A$ Similarly, the vector fields $X_{2}$ and $X_{3}$ in Eq (27) yield to one-soliton solutions of the same form as in $\mathrm{Eq}(61)$, with $v_{1}(K)$ replaced, respectively, by 
$v_{2}(K)=32 \sinh (4 K)+128 \sinh (2 K)$ and $v_{3}(K)=128 \sinh (6 K)+768 \sinh (4 K)+$ $1920 \sinh (2 K)$

We pass to the interpolated KM system (in the sense of Sect 3), and consider the vector fields $X_{s}(s=1,2,3)$ For each choice of the lattice spacing $\varepsilon$, we can construct one-soliton solutions for these vector fields, replacing the index $i$ in Eq. (6.1) with the ratio $x / \varepsilon$, the parameters $K$ and $A$ in the above equation can be chosen arbitrarily for any given $\varepsilon$. If we make the choices $K=\varepsilon k$ (with $k$ a real constant) and keep $A$ independent of $\varepsilon$, we are led to the solutions

$$
a^{\varepsilon}\left(x, t_{\varsigma}\right)=2 \sqrt{\frac{\cosh (3 \varepsilon k)+\cosh 2\left[k(x-\varepsilon / 2)+v_{s}^{\prime}(k) t_{\varsigma}+A\right]}{\cosh (\varepsilon k)+\cosh 2\left[k(x-\varepsilon / 2)+v_{\varsigma}^{\prime}(k) t_{\varsigma}+A\right]}}
$$

Here, $k$ and $A$ are arbitrary, while

$$
\begin{aligned}
& v_{1}^{\prime}(k)=8 \sinh (2 \varepsilon k), \quad r_{2}^{\prime}(k)=32 \sinh (4 \varepsilon k)+128 \sinh (2 \varepsilon k), \\
& r_{3}^{\prime}(k)=128 \sinh (6 \varepsilon k)+768 \sinh (4 \varepsilon k)+1920 \sinh (2 \varepsilon k)
\end{aligned}
$$

In the previous two sections we have shown how to construct from the vector fields $X_{s}^{*}$ the recombinations $Z_{\xi}^{\xi}$, in such a way that the ratio $Z_{\xi}^{\xi} / \varepsilon^{2 s-1}$ gives, in the $\varepsilon \mapsto 0$ limit, the vector field $4^{\mathrm{s}+1} Z_{\uparrow}^{\mathrm{KdV}}$, in particular, the first three recombinations are as in $\mathrm{Eq}(413)$ Let us use again the field variable $a(x)$ rather than the rescaled field $u(x)$ of $\mathrm{Eq}(41)$, which will appear at the end of this computation, for $s=1,2,3$, we consider the evolution equations $d a / d \tau_{\varsigma}=Z_{s}(a) / \varepsilon^{2 s-1}$, which admit solutions of the same form as in $\mathrm{Eq}(6.2)$, with ${ }_{s}$ replaced by $\mu_{s}$, where

$$
\begin{aligned}
& \mu_{1}^{i}(k)=\frac{1}{\varepsilon} v_{1}^{i}(k), \quad \mu_{2}^{\varepsilon}(k)=\frac{1}{\varepsilon^{3}}\left(v_{2}^{\varepsilon}(k)-24 v_{1}^{\varepsilon}(k)\right), \\
& \mu_{3}^{\prime}(k)=\frac{1}{\varepsilon^{5}}\left(v_{3}^{\prime}(k)-40 v_{2}^{\prime \prime}(k)+480 v_{1}^{\prime}(k)\right)
\end{aligned}
$$

Clearly, the preservation of the form $(62)$ for the solutions, modulo a recombination of $v_{1}^{\prime}, v_{2}^{\prime}$ and $v_{3}^{\prime}$ with the same coefficients as for the vector fields, is a consequence of the commutativity of the flows under examination.

The final step in our considerations is the $s \mapsto 0$ limit of the solutions

$$
a^{i}\left(x, \tau_{\varsigma}\right)=2 \sqrt{\frac{\cosh (3 \varepsilon k)+\cosh 2\left[k(x-\varepsilon / 2)+\mu_{s}^{i}(k) \tau_{s}+A\right]}{\cosh (\varepsilon k)+\cosh 2\left[k(x-\varepsilon / 2)+\mu_{s}^{i}(k) \tau_{s}+A\right]}}
$$

It is easily checked that

$$
\begin{gathered}
\mu_{1}^{i}(k)=16 k+\frac{32}{3} \varepsilon^{2} k^{3}+O\left(\varepsilon^{3}\right), \quad \mu_{2}^{i}(k)=256 k^{3}+256 \varepsilon^{2} k^{5}+O\left(\varepsilon^{3}\right), \\
\mu_{3}^{i}(k)=4096 k^{5}+\frac{16384}{3} \varepsilon^{2} k^{7}+O\left(\varepsilon^{3}\right)
\end{gathered}
$$

Inserting these expansions in Eq. (6 5) and developing again up to $O\left(\varepsilon^{3}\right)$, we finally obtain (for $s=1,2,3$ )

$$
a^{i}\left(x, \tau_{\varsigma}\right)=2+\varepsilon^{2} \frac{2 k^{2}}{\cosh ^{2}\left(k x+16^{\prime} k^{2 \varsigma-1} \tau_{\varsigma}+A\right)}+O\left(\varepsilon^{3}\right)
$$


Passing to the rescaled field variable $u$, as in Eq. (4.1), we see that the function $u^{\prime \prime}\left(x, \tau_{s}\right)=\left(1 / \varepsilon^{2}\right)\left(a^{i}\left(x, \tau_{s}\right)-2\right)$ converges, for $\varepsilon \mapsto 0$, to the familiar one-soliton solution for the vector field $4^{s+1} Z_{\varsigma}^{\mathrm{KdV}}$ A similar analysis of the one-soliton solutions and their zero-spacing limit could be performed for the higher order vector fields, with $s>3$

After this example, we return to the general problem outlined in this section Setting up general convergence results for the solutions (with arbitrary initial data) is a problem that should be attacked with appropriate tools One should prove (for all values of $s$ ) that any solution $u\left(x, \tau_{s}\right)$ of the evolution equation $d u / d \tau_{s}=$ $Z_{\varsigma}^{\mathrm{KdV}}(u)$ is the $\varepsilon \mapsto 0$ limit of a solution $u^{\varepsilon}\left(x, t_{\varsigma}\right)$, corresponding to the vector field $Z ; / \varepsilon^{2 i-1}$

In connection with this topic, we mention the rigorous results obtained in [Sch] on the continuous limit of the KM vector field $X_{1}$ In this paper, Schwarz discusses the $\mathrm{KdV}$ equation $(11)$ as a limit of $X_{1}$, using a space-time coordinate change of the form (4 24) (incidentally, he also reports a computation of Kac on the limit of the one-soliton solutions for $X_{1}$, based on the above transformation) The beautiful convergence results of Schwarz are stated in the framework of Sobolev spaces, and concern the solutions of the Cauchy problem with arbitrary initial data

Apart from the specification of the analytical setting, there are some technical differences between our approach and the one of Schwarz Concerning the derivations of Eq ( 11 ), we repeat the comment following Eq (4 24) from our viewpoint, the $\mathrm{KdV}$ evolution equation $\left(\begin{array}{ll}1 & 1\end{array}\right)$ does not come from $X_{1}$ through a space-time transformation, but from a recombination of $X_{2}$ and $X_{1}$ Another difference is that Schwarz does not explicitly introduce an analogue of our "interpolating KM system," with a projection $\Pi^{\delta}$ onto the discrete system as explained in the Remark at the end of Sect 3 , rather, he gives a constructive prescription to interpolate a sequence $\left(a_{i}\right)$ with a function $a(x)$ (which amounts, in geometrical terms, to selecting a particular right inverse of our projection $\Pi^{*}$ )

In spite of the above facts, we think that Schwarz' method could be implemented into our scheme, modulo the necessary embedding of our setting into the rigorous framework of the Sobolev chains In fact, the essential point in the convergence theorems of $[\mathrm{Sch}]$ is the possibility to obtain a priori bounds (uniformly in 6 ) on some conveniently defined differential-difference analogues of the Sobolev norms of $u(\cdot, \tau)$ These bounds on the solutions are derived using the functionals $h_{\text {, }}$, which are constants of motion for the vector field $X_{1}$ under consideration on the other hand, the $h$,'s or their recombinations are conserved quantities for the full $\mathrm{KM}$ hierarchy, so the technique proposed for $X_{1}$ can probably be extended to treat the higher order vector fields

Schwarz employs the estimates on $X_{1}$ to infer a priori estimates for the solutions of the $\mathrm{KdV}$ equation, in the ordinary Sobolev norms, such a priori bounds mean that the solutions of the $\mathrm{KdV}$ equation do not develop shocks As a final remark, we note that there are cases, known from the literature, in which the limiting system of an integrable lattice develops shocks, depending on the procedure that defines the limit In particular, Bloch and Brockett [BB] have illustrated a limit process for the Toda lattice, yelding to a system of hydrodynamic type in which a shock phenomenon occurs 
This paper has been partially supported by Consiglio Nazionale delle Ricerche, G N F M, and by Ministero dell'Università e della Riceıca Scientifica, Project "Metodi Geometı ici e Probabilistici in Tcorie di Campo"

\section{References}

[BB] Brockett, R W, Bloch, A : Sorting with the dispersionless limit of the Toda lattice Proceedings of the CRM Workshop on Hamiltonian systems, transformation groups and specthal transform methods, J Harnad, JE Marsden, (eds), 1990, pp 103-112

[CCK] Case, KM: On discrete inverse scattering problems II J Math Phys 14, 916-920 (1974); Case, K M, Kac, M: A discrete version of the inverse scattering problem J Math Phys 14, 594-603 (1974); Case, KM, Chiu, S C: The disciete version of the Marchenko equations in the inverse scattering problem $J$ Math Phys 14, 1643-1647 (1973)

[Dam] Damianou, P A : Multiple Hamiltonian structures for Toda-type systems J Math Phys 35, 5511-5541 (1994)

[Dic] Dickey, L A : Soliton equations and Hamiltonian systems Singapore: World Scientific, 1991

[DS] Dinfeld, V G, Sokolov, V V : Lie algebras and equations of Korteweg-de Vries type J Sov Math 30, 1975-2036 (1985)

[Fla] Flaschka, H: The Toda lattice I Phys Rev B 9, 1924-25 (1974)

[GJ] Glimm, J, Jaffe, A : Quantum Physics A functional integral point of view Berlin: Springer-Verlag, 1981

[KM] Kac. M, van Moerbeke, P: On some periodic Toda lattices Proc Nat Acad Sci USA 72, 1627-1629 (1975); On an explicitly soluble system of nonlinear differential equations 1clated to certain Toda lattices Adv in Math 16, 160-169 (1975)

[Kup] Kupershmidt, B A : Discrete Lax equations and differential-difference calculus Astérisque 123 (1985), Soc Math de France

[Mag] Magi i, F : A simple model of the integrable Hamiltonıan equation J Math Phys 19, $1156-1162(1978)$

[Man] Manakov, S V : Complete integrability and stochastization of disciete dynamical systems Sov Phys JETP 40, 269-274 (1975)

[MMR] Magı, F, Morosi, C, Ragnisco, O : Reduction techniques for infinite-dimensional Hamiltonian systems Some ideas and applications Commun Math Phys 99, 115-140 (1985)

[Sai] Saitoh, N : A transformation connectıng the Toda lattice and the KdV equation J Phys Soc Japan 49, 409-416 (1980); The Bäcklund tıansformation and Hamiltonian formalism for a generalized Toda lattice J Phys Soc Japan 54, 3261-3267 (1985)

[Sch] Schwaiz, M : Kolteweg-de Vries and nonlineal equations related to the Toda lattice Adv in Math 44, 132-154 (1982)

[Tod] Toda, M: Theory of nonlinear lattıces (2nd Enlarged Edition) Berlin: Springer-Verlag. 1989

[TW] Toda, M, Wadatı, M : A soliton and two solitons in an exponential lattice and related equations J Phys Soc Japan 34, 18-25 (1973)

[ZK] Zabusky, N J, Kıuskal, M D: Interaction of "solitons" in a collisionless plasma and the recurrence of initial states Phys Rev Lett 15, 240-243 (1965)

[ZMR] Zakharov, VE, Mushes, S L, Rubenchik. A M : Nonlinear stage of parametric wave excitation in a plasma JETP Lett 19, 151-152 (1974) 